\title{
A microprocessor-based psychopathology laboratory: II. Research goals and design prerequisites
}

\author{
WILL SPAULDING and LAWRENCE G. SPACE \\ University of Rochester, Rochester, New York 14627
}

\begin{abstract}
The design of a microprocessor-based psychopathology laboratory is influenced by (1) special procedures in many experimental paradigms and (2) some theoretical considerations about psychopathological data. In terms of design prerequisites, these are summarized as flexibility and power to control a spectrum of experiments, large data-processing capacity, ability to provide clinically relevant data on short notice, mobility, and simplicity of operation and programming.
\end{abstract}

The experimental procedures involved in our research determine many of the laboratory's design prerequisites. Also, there are some theoretical points that have important implications for how psychopathological data should be collected and interpreted. These implications have also influenced the laboratory's design.

\section{THE INFORMATION-PROCESSING MODEL}

Cognition is complex. Relationships between $a b$ normal cognition and psychiatric symptoms are more so. One way of dealing with this is to associate specific symptom patterns with abnormalities in specific components of the cognitive system. The familiar threestage model of cognition diagrammed in Figure 1 provides a structure for defining components of the system.

Cognition in this model is represented as information proceeding through registers. The registers and associated transfer operations are grouped into the three components diagrammed. The short-term memory (STM) component of this model received much initial attention in psychopathology. Many STM processes were found to be deficient, primarily in schizophrenics. However, conclusions from those studies were predicated on an intact icon. Unfortunately, most experimental techniques for measuring icons require an intact STM. One experiment, developed by Erikson and Collins (1967), put minimal demands on STM and provided a measure of iconic functioning.

\section{ICON FUNCTIONING}

When two sequential visual stimuli occur during a short enough time frame, they are summed, or entered on the same icon. To measure the required time frame, two dot patterns are sequentially presented in a tachisto.

Acknowledgment is made to NIMH Grant MH-14650 and New York State Health Research Council Grant HRC 846. scope. The two patterns together form a two-digit number (Figure 2). The subject can report the number only if summation has occurred. Varying the time interval between stimuli produces a psychophysical function which reflects an operating characteristic of the icon.

In normal subjects, report of the digits decreases rapidly when the asynchrony is greater than a few milliseconds. Hospitalized patients generally have a faster decay of summation over time (see Figure 2). However, the abnormality is not characteristic of a particular type of patient. This may reflect the low SES, low IQ, or high anxiety of inpatients, so some further investigation is indicated.

The speed with which information is transferred between the icon and STM can be measured with back ward masking. A mask is a stimulus that follows a target stimulus so closely in time that it "erases" the target's

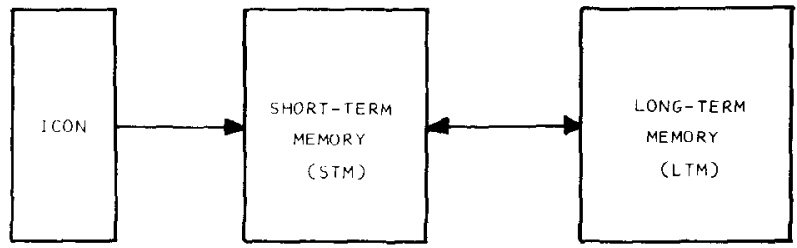

Figure 1. The three-stage information-processing model of cognition.
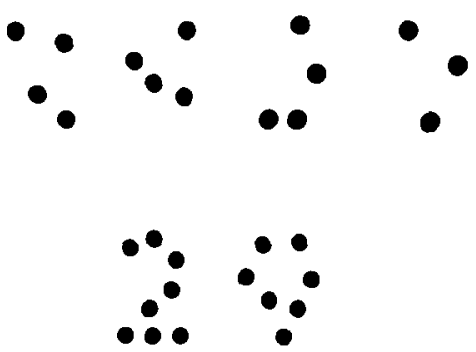

Figure 2. Two stimuli presented sequentially (above); if summated, they form the two-digit number (below). 


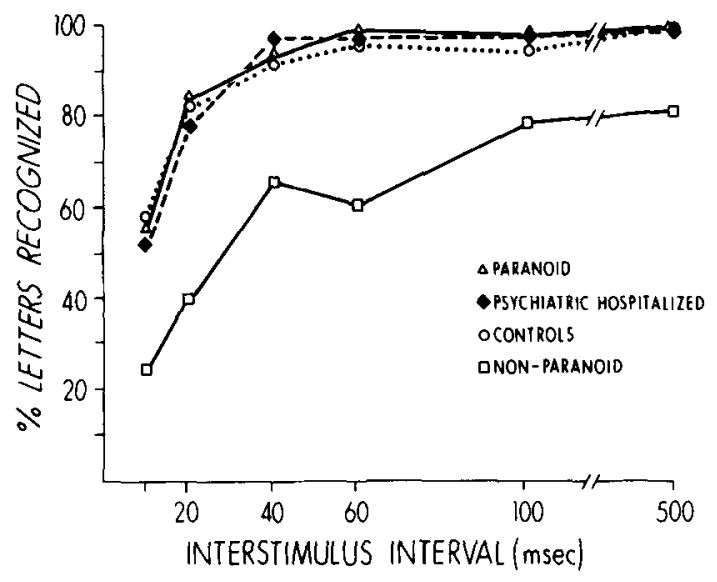

Figure 3. Masking function in psychiatric groups and normals. From Freedman (1977) by permission of the author.

icon. By flashing the mask at varying delays, the researcher controls the time STM has access to the target icon. Figure 3 shows report of a two-letter target in normals and various patient groups (Freedman, 1977). The slope interaction of these curves is significant; the low group did not reach asymptote at the last interval, as the other groups did. Therefore, it is difficult to account for these differences in terms of global motivational or intellectual factors. Rather, the nonparanoids seem to have abnormally slow encoding.

\section{STM FUNCTIONS}

Some processes perform the important function of restricting input to the STM registers. Further, the inputreceiving capacity of STM is greater than its subsequent processing capacity. Therefore, channel-selection processes are required to determine which channel must be further processed. When these processes fail, that is, when irrelevant information or noise enters STM registers, distraction has occurred.

Distractibility and the effects of distraction are closely associated with psychophysiological variables (Tecce \& Cole, 1976). This is especially interesting to psychopathologists because some patient groups show characteristic psychophysiological abnormalities as well as distractibility.

The psychophysiology of distractibility may be a key to understanding phasic deficits (those that are not present all the time) in STM. In a realistic social situation, it is much easier to measure psychophysiology than it is to directly measure distractibility with a $\mathrm{T}$-scope type procedure. Through in vivo monitoring of psychophysiology during social interaction, inferences may be drawn about phasic cognitive deficits by observing phasic psychophysiological abnormalities.

The manipulation of information within STM can be measured in psychiatric patients with an adaption of Estes' "forced-choice span of apprehension" paradigm
(Neale, 1971). The subject sees a matrix of letters very briefly (about $100 \mathrm{msec}$ ) in a tachistoscope. The matrix contains one to two target letters. The subject must report which of the two was present.

To perform this task, one must sequentially analyze features of the letters until a decision can be reached. There is time to construct only one icon of the matrix, so STM must do the job in about $500 \mathrm{msec}$. By applying a mathematical formula to performance over many trials, the average number of letters processed per trial is calculated.

Process schizophrenics appear to be inefficient when matrix size is four or more letters (Neale, 1971). In view of data from the backward masking studies, revealing deficits in the same patient group, performance in the span of apprehension paradigm may reflect encoding difficulties rather than data manipulation deficits within STM.

Another STM process is information chunking. This is represented by the use of concepts. In the informationprocessing models, concepts are response-relevant codes which must be manipulated to achieve response selection.

Figure 4 illustrates a test that measures one aspect of conceptual manipulation in STM. The figures in each upper box represent three-way combinations of three concepts-texture, form, and number. In the lower box a figure is presented from a pool of all possible combinations. The task is to sort each figure that appears in the lower box into one of the three upper boxes, depending on which concept is relevant. The experimenter sets the relevant concept and gives positive or negative feedback for each trial. The subject must figure out the correct concept through trial and error. After five correct sorts, the criterion shifts to another concept. The subject must figure out from the feedback that the criterion has changed and must modify responses accordingly.

Paranoid patients have particular difficulty on this task (Fey, 1952; Spaulding, 1978). Once they succeed with one criterion, they are extremely hesitant to change, even with repeatedly negative feedback.

\section{LTM STRUCTURES}

In realistic situations, the concepts used in STM are determined in large part by information stored in

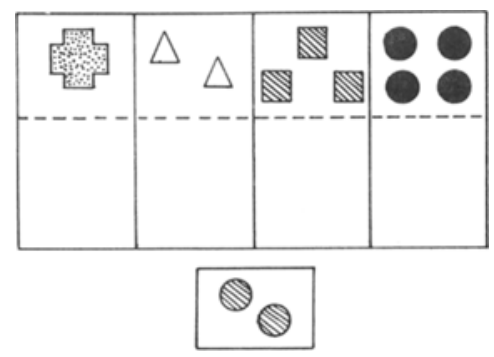

Figure 4. Format of a conceptual modulation test. 
long-term memory (LTM). These stored structures include attitudes, beliefs, and similar strategies for simplifying complex information. It has long been known that psychiatric patients often have peculiar structures in LTM. These structures have been systematically studied with nomothetic instruments like the MMPI, and less systematically with ideographic approaches such as word association and projective techniques. The Kelly role construct repertory grid technique provides a more fine-grained analysis of individual LTM structures. Instead of relying on nomothetic dimensions, the rep grid evokes the subject's own concepts, attitudes, and beliefs. This is accomplished by having the individual provide names of significant people in his or her environment. The names are then presented back to the subject in various combinations. The subject compares and contrasts the groups of names and reports the personal descriptors used to do so. The descriptors then serve as labels for scalar dimensions. The subject rates the people previously named on each dimension, producing a matrix or grid. The grid may then be factor analyzed, producing a kind of map of the subject's conceptual structure.

Quantitative analysis of the rep grid structure has been useful in identifying specific abnormalities in conceptual structures (Space, 1976; Space \& Cromwell, 1978). Also, qualitative analysis can identify what aspects of conceptual structure should be modified in psychotherapy. The procedure required to obtain this data is extremely cumbersome, so the rep grid is a particularly attractive candidate for computer automation.

\section{SOME THEORETICAL CONSIDERATIONS}

Information-processing abnormalities must be understood in the context of individuals' development and current social environment. Specific deficits are associated with various other factors. Cause-and-effect relationships between cognition, behavior, physiology, and social environment can seldom be determined solely with laboratory data. Psychopathology has in the past been hampered by inadequate attention to such factors. When appropriate attention is given, certain crucial facts emerge: (1) Some cognitive deficits are tonic (i.e., present all the time) and others are phasic. As mentioned, psychophysiological factors are often involved. (2) Some deficits are the product of constitutional factors, others of early learning, others of more recent and situation-specific learning. (3) Some deficits produce specific symptoms of psychosis. Others produce subclinical abnormalities that interact with other factors to produce psychosis. The latter are termed vulnerabilitylinked deficits. In some cases, vulnerability may be genetically linked. Asarnow, Steffy, MacCrimmon, and Cleghorn (1978) found that span of apprehension deficits appear in remitted schizophrenics and children of schizophrenic parents. De Amicis (1977) found that a particular reaction time pattern associated with process schizophrenics also occurred in nonschizophrenic members of patients' families. (4) Some deficits produce other deficits. Direction of causality may become obscured. For example, distractibility may increase arousal, which increases sensitivity to distraction, and so on. (5) There are specific patterns of cognitive deficit that are characteristic of specific behavioral and historical patterns. However, these relationships are imperfect. A specific cognitive deficit may produce many possible behavior patterns, and a specific behavior pattern may be the result of various deficits.

The complexity of these relationships is illustrated in Figure 5. The figure is a diagrammatic representation of a series of regression analyses performed by Spaulding (1978). The circles on the left (independent variables) represent laboratory measures of four levels in the information-processing system. On the right are clinical indexes of abnormal behavior. The numbers associated with the unidirectional arrows are standardized multipleregression coefficients. The bidirectional arrow represents a simple correlation between two predictor variables.

These facts have several implications. First, psycho-

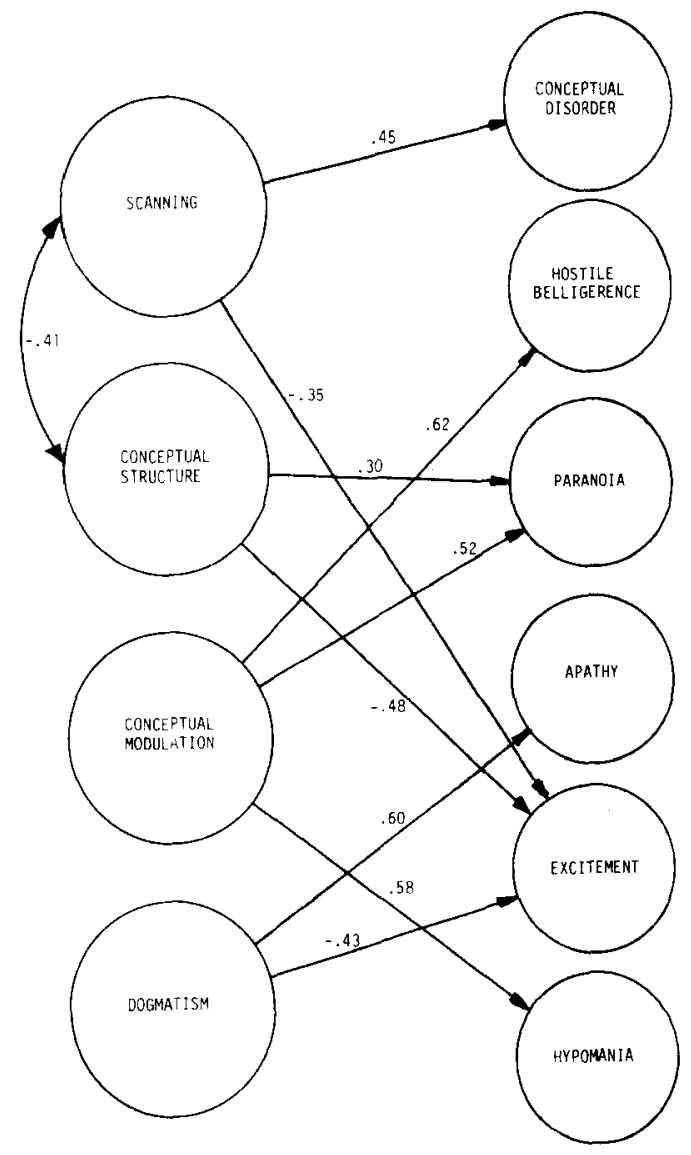

Figure 5. Interrelationships of cognitive abnormalities and psychiatric symptoms. 
pathologists must have access to heterogeneous patient populations. They must count on finding a spectrum of meaningful abnormalities. They must gather considerable amounts of clinical and historical data to interpret laboratory data. All this requires a close working relationship with clinical personnel. The relationship is facilitated if the psychopathology lab can provide meaningful information to clinical staff, to assist in assessment and treatment.

There are other reasons for providing treatmentrelevant data. One is, of course, financial. Clinical work provides relatively stable income and may even support research when grant funds are reduced. Another is the opportunity to generate time series data on treatment response. For all these reasons, one goal of our research has been to develop a subset of paradigms that provide data with relatively immediate clinical implications.

The vulnerability concept generates a special demand. In order to study vulnerability linked deficits, nonpsychotic subjects are needed. These are often family members of inpatient index subjects. Psychiatric patients' families tend to be less cohesive, geographically scattered, and less than willing to participate in research. Psychopathologists must be prepared to gather data aggressively outside the home lab.

\section{SUMMARY}

All these considerations can be summarized in terms of design prerequisites of a computerized psychopathology laboratory: (1) Flexibility and power to handle the broad spectrum of experimental paradigms involved. This means maximizing central processing capabilities and minimizing cost for specialized peripheral equipment. (2) Capacity to store large amounts of laboratory and other data. Data preferably will be stored in raw form, to permit a wide range of subsequent analyses. (3) Ability to provide clinically relevant information on short notice, in other words, to be accessible to some degree to clinical personnel. (4) Mobility to gather data at distant locations or in in vivo settings. (5) Simplicity of operation to allow rapid modification of experiment control and data-manipulation routines. Programming at this level must be possible for personnel with modest computer skills.

\section{REFERENCES}

Asarnow, R., Steffy, R., MacCrummon, D., \& Cleghorn, J. An attentional assessment of foster chidren at risk for schizophrenia. In L. Wynne, R. Cromwell, \& W. Mathysse (Eds.), The nature of schizophrenia. New York: Wiley, 1978.

De Amicis, L. A study of crossover in the reaction time performance of process schizophrenics and their first-degree relatives. Unpublished doctoral dissertation, University of Rochester, 1977.

ERIKson, C., \& Collins, J. Some temporal characteristics of visual pattern perception. Journal of Experimental Psychology, $1967,74,476-484$.

FEY, E. The performance of young schizophrenics on the Wisconsin Card Sorting Task. Journal of Consulting and Clinical Psychology, 1952, 15, 311-319.

Freedman, J. Backward masking in paranoid and nonparanoid schizophrenics and in normals. Unpublished master's thesis, University of Arizona, 1977.

NeALE, J. Perceptual span in schizophrenia. Journal of Abnormal Psychology, 1971, 77, 196-204.

SPACE, L. Cognitive structure comparison of depressives, neurotics, and normals. Unpublished doctoral dissertation, Wayne State University, 1976.

SPACE, L., \& CROMwell, R. Personal constructs among schizophrenics. In S. Schwarz (Ed.), Language and cognition in schizophrenia. New York: Erlbaum, 1978.

SPAUlding, W. The relationships of some information processing factors to severely disturbed behavior. Journal of Nervous \& Mental Disease, 1978, 166, 417-428.

Tecce, J., \& Cole, J. The distraction-arousal hypothesis, CNV, and schizophrenia. In D. Mostofsky (Ed.), Behavior control and modification of physiological activity. Englewood Cliffs, N.J: Prentice-Hall, 1976. 